\title{
Atomic pectroscopy \\ Signal Enhancement in Spark-assisted vs. Dual-beam Laser-induced Breakdown Spectroscopy
}

\author{
Mahdi Habibpour, Parviz Parvin, ${ }^{*}$ Reza Amrollahi, and Shirin Gholami \\ Physics Department, Amir Kabir University of Technology, P.O. Box 15875-4413, Tehran, Iran
}

Received: March 26, 2021; Revised: April 12, 2021; Accepted: April 12, 2021; Available online: April $20,2021$.

DOI: $10.46770 / A S .2021 .023$

ABSTRACT: In this work, the characteristic emission lines of $\mathrm{Cu}, \mathrm{Au}, \mathrm{Ag}, \mathrm{Al}, \mathrm{Ni}$, and $\mathrm{Pd}$ are obtained in a Q-switched Nd: YAG laser-induced plasma. Furthermore, the signal enhancement is carried out separately by making use of the continuous-wave (CW) $\mathrm{CO}_{2}$ laser as well as the spark discharge. The former enhances the signal intensity via plasma heating according to the inverse Bremsstrahlung (IB) mechanism, whereas the latter relies on plasma reheating and subsequent lifetime elongation. As a consequence, both methods lead to the temperature rise of the plasma. In addition, the corresponding electron density of the plasma is notably elevated in sparkassisted LIBS (SA-LIBS). It is also shown that the enhancement of the ionic lines is much higher in SA-LIBS against the other one. Although the $\mathrm{CW}-\mathrm{CO}_{2}$ laser benefits an electrodeless arrangement, SA-LIBS is considered to be a better candidate due to its facile and low-cost setup.

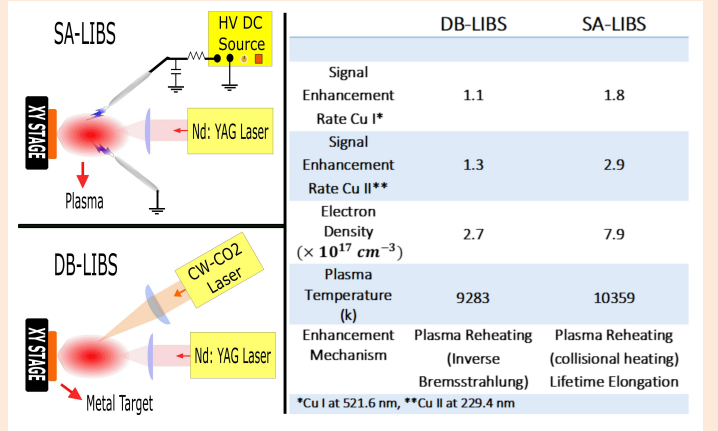

\section{INTRODUCTION}

Laser-induced breakdown spectroscopy (LIBS) is an analytical technique based on atomic emission spectroscopy. Applying a high-power laser beam on the target and delivering enough energy, the evaporation and atomization of the sample will simultaneously occur, which induces a small crater on the surface. The ionized vapor emission consists of atomic and ionic spectral lines proportional to the elemental compositions. The advantages of LIBS with respect to conventional analytical techniques include robustness, fast response, and high sensitivity (generally in ppm range), which makes it attractive for applications where fast multicomponent analysis is required. ${ }^{1,2}$ A variety of samples with any shape can be analyzed by implementing this method without the necessity of an analytical chamber or surface treatment, and recently its portable version is also available. ${ }^{3}$ Furthermore, the capability of applying LIBS for remote sensing without sample preparation makes it a reasonable choice in any environment, from on-line measurements in industry to space exploration. ${ }^{4,5}$ In addition, for quantitative analysis, the calibration-free (CF) technique coupled with LIBS has been developed. Based on three main hypotheses, i.e. local thermal equilibrium (LTE), homogeneous ablation, and optically thin plasma, Ciucci et al. ${ }^{6}$ developed a calibration-free technique that requires no external calibration standards presuming that all species contained in the sample are detected. This method has also been successfully applied for the compositional analysis of oxide materials, ${ }^{7}$ food, ${ }^{8}$ metallic alloys, ${ }^{9-12}$ and soils. ${ }^{13,14}$

On the other hand, over the last two decades many attempts have been made to improve the sensitivity and the limits of detection of LIBS. Current effective approaches, including spatial or magnetic confinement, ${ }^{15,16}$ double or multiple-pulse excitation, ${ }^{17,18}$ microwave emission, ${ }^{19}$ glow or spark discharge,${ }^{20,21}$ and laser-induced fluorescence and resonance enhancement, ${ }^{22,23}$ have been reviewed in addition to other methods by Li et al. ${ }^{24}$ Most of these techniques rely on plasma reheating and lifetime elongation by applying an additional excitation source. Killinger et al. ${ }^{25}$ used a simultaneous $\mathrm{CO}_{2}$ laser pulse at $10.6 \mu \mathrm{m}$ to enhance the LIBS emission on a high purity alumina target. The enhancement factor on the order of 25 to 300 times was achieved, depending on the emission lines. They found that the timing overlap of the dual laser pulses within 1 microsecond is critical to attain the enhancement factor. Shoursheini et al. ${ }^{26}$ investigated 
enhancement of the $\mathrm{Cu}$ emission lines in LIBS due to the thermal effect of simultaneous irradiation of a Q-switched Nd: YAG and a $\mathrm{CW}-\mathrm{CO}_{2}$ laser. In order to avoid distortion of the samples, the focal point of the laser was located $\sim 1 \mathrm{~mm}$ above the surface to generate the plasma in the air rather than on a solid surface. They noted that the far infrared laser irradiation preheats the sample, leading to a rise in plasma temperature. Nassef et al. ${ }^{27}$ combined LIBS with a spark discharge operating in a laser-trigger mode using $\mathrm{Al}$ and $\mathrm{Cu}$ targets in the air under atmospheric pressure. They found that the enhanced spectral intensity and signal-tobackground (S/B) ratio in spark-assisted (SA)-LIBS gives rise to a significant reduction in the required laser energy against the standard LIBS. The fundamentals, instrumentation, development, and applications of the SA-LIBS technique were reviewed by Zhou et al. ${ }^{28}$ Recently, high repetition rate laser ablation sparkinduced breakdown spectroscopy was implemented combined with the CF method for the quantitative elemental analysis of aluminum and silver alloys. ${ }^{29,30}$ Eventually, single-shot SA-LIBS was successfully used for gold fineness assessment based on the CF method. ${ }^{31}$

In this study, the characteristic line intensification of a Qswitched Nd: YAG laser-induced plasma is investigated in dualbeam LIBS (DB-LIBS) and SA-LIBS arrangements employing a $\mathrm{CW}-\mathrm{CO}_{2}$ laser and a spark discharge, respectively. These methods enhance plasma emission by elevating the plasma temperature. According to the inverse Bremsstrahlung mechanism, DB-LIBS enhances the signal intensity via plasma heating, whereas SALIBS relies on plasma reheating and lifetime elongation. Regarding the plasma electron density measurements, the LTE criteria are strongly satisfied in SA-LIBS due to its higher electron number density. Although the $\mathrm{CW}-\mathrm{CO}_{2}$ laser benefits an electrodeless arrangement, SA-LIBS is better when used in portable devices in terms of cost and compactness.

\section{EXPERIMENTAL}

Standard LIBS. Fig. 1 illustrates the experimental setup, consisting of a laser source, focusing and conducting optics, spectrometer, high voltage power supply, PIN diode, voltage and current probes, and a digital oscilloscope. A master oscillatoramplifier Q-switched Nd: YAG laser (1064 nm, $10-100 \mathrm{~mJ}$, 10-ns duration, $1-10 \mathrm{~Hz}$ ) was used as a coherent source. A Coherent joule meter (Field Master, LM-P10, and LM-P5100 heads) was employed for the absolute pulsed energy measurements of the laser shots. The laser beam was focused on the metal target at an angle of 45 degrees with respect to the sample surface using a plano-convex lens with a $20 \mathrm{~cm}$ focal length. The target was located at a position of $18 \mathrm{~cm}$ far from the lens L1 so that the laser beam is focused below the sample surface. Subsequently, maximum peak power of approximately $10 \mathrm{MW}$ was obtained for a single shot, delivering $\sim 30 \mathrm{GW} / \mathrm{cm}^{2}$ at the sample surface for a 200- $\mu \mathrm{m}$ diameter spot (measured using a

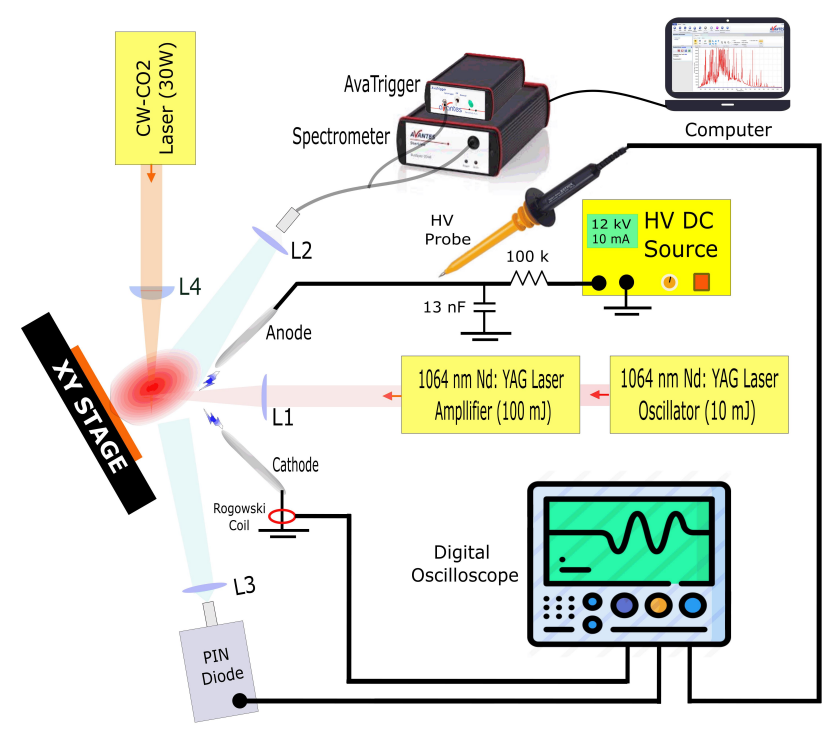

Fig. 1 DB-LIBS and SA-LIBS setup, including Q-SW Nd: YAG laser, $\mathrm{CW}-\mathrm{CO}_{2}$ laser, spark generator circuit, digital oscilloscope, spectrometer, electrodes, HV probe, Rogowski coil, and focusing lenses L1, L2, L3, and L4.

microscope). A commercial collimating quartz lens L2 of $6 \mathrm{~mm}$ diameter and $8 \mathrm{~mm}$ focal length was placed $50 \mathrm{~mm}$ away from the sample and aligned at an angle of 45 degrees with respect to the direction of the laser beam. This forms an image of plasma on the input of a fiber bundle (Avantes, FC-UVIR200-1). The fiber output was coupled to the slit of a compact wide-range spectrometer (Avantes, AvaSpec 2048, 200-1100 nm, $0.4 \mathrm{~nm}$ precision), where it is externally triggered using an AvaTrigger to start signal integration with a minimum delay time of $1.2 \mu \mathrm{s}$ with an integration time of $1.1 \mathrm{~ms}$. A semiconductor detector (PIN diode, EG\&G, FNT100) was used to detect the optical signal intensity through a lens L3. The electric signals were also simultaneously monitored with a digital storage oscilloscope (Good Will, GDS-1054B). Different pure samples (99.99 \%), i.e., $\mathrm{Au}, \mathrm{Cu}, \mathrm{Ag}, \mathrm{Ni}, \mathrm{Al}$, and $\mathrm{Pd}$, were chosen as targets during the experiments. The target foil was mounted on a motorized twodimension $\mathrm{x}-\mathrm{y}$ moving stage to expose a fresh spot for each trial. The target's surface was manually polished using 150-grit fine sandpaper before the next shot to provide nearly identical exposure conditions. The spectral measurements were repeated over 10 shots to obtain the relative standard deviation (RSD) values.

Dual-Beam LIBS. A home-made sealed CW-CO 2 laser beam at $10.6 \mu \mathrm{m}$ with $5 \mathrm{~mm}$ diameter and mean power of up to $30 \mathrm{~W}$ was focused on the target. A ZnSe lens L4 with $150 \mathrm{~mm}$ focal length was situated in front of the beam to scale up the power density. Before the Nd: YAG laser shot, the $\mathrm{CO}_{2}$ laser beam is irradiated on the sample, while the focal point was located $3 \mathrm{~mm}$ above the target surface. The surface temperature was measured using a calibrated Fluke $52 \mathrm{II}$ thermometer with $0.1^{\circ} \mathrm{C}$ resolution. 

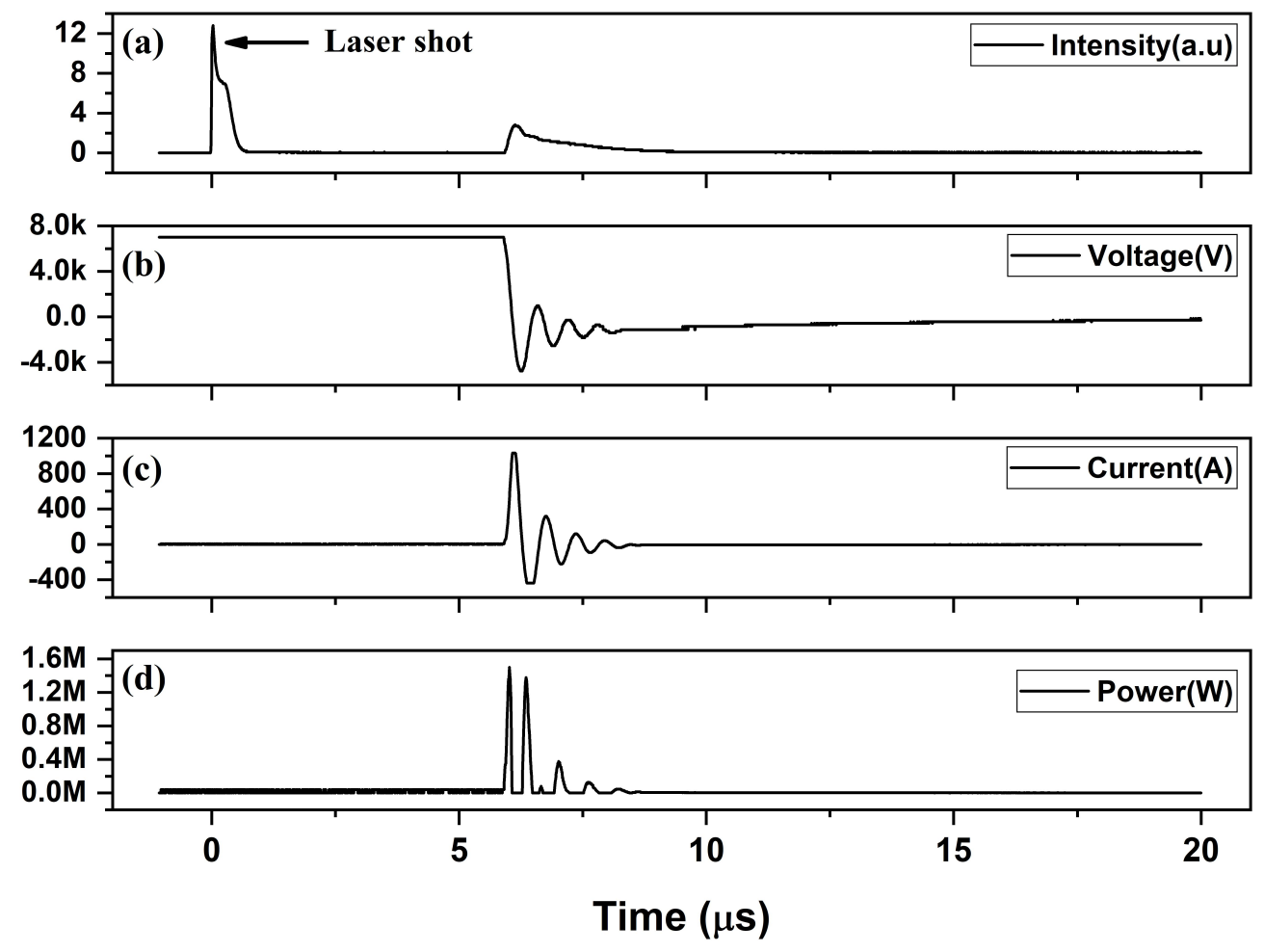

Fig. 2 Optical and electrical signals during a typical SA-LIBS experiment. The voltage output of (a) PIN diode, (b) H.V probe, (c) Rogowski coil, and (d) the multiplication of $v(t) \times I(t)$ addressing the discharge power. The zero position on the $\mathrm{x}$-axis corresponds to the onset of the laser shot.

Spark Generator. The spark generator consists of a high voltage power supply and a pair of electrodes made of stainless steel in the form of cylindrical rods, $2 \mathrm{~mm}$ in diameter, with a curve-shaped tip. The gap between the electrodes was set to be $6 \mathrm{~mm}$ which is located $5 \mathrm{~mm}$ far from the target surface, in order to avoid the electric self-breakdown at $7 \mathrm{kV}$. The sample was electrically insulated from the stage. A home-made variable power supply $(1-12 \mathrm{kV} ; 10 \mathrm{~mA}$ ) was fabricated to generate the spark discharge. The laser irradiation ignited the electric breakdown between the electrodes via the electron injection into the middle of the gap. The voltage shape was measured using a Pintek 15-HF high voltage probe, and the current was monitored by means of a calibrated Rogowski coil accordingly.

\section{RESULTS AND DISCUSSION}

Discharge characteristics. Fig. 2 depicts the temporal optical and electrical signals of a typical experiment where the inter-pulse delay is set to be $6 \mu \mathrm{s}$. The PIN photodiode signal undergoes a peak when the laser is triggered and elevates once again after $6 \mu \mathrm{s}$ following the occurrence of the peak current and smoothly decays at a lower rate (Fig. 2a). At the onset of the electric breakdown, the voltage across the capacitor drops rapidly from $7 \mathrm{kV}$ to zero (Fig. $2 b$ ), and the current rises to $1 k A$ passing through the plasma (Fig. 2c). As a result, the discharge peak power reaches $1.5 \mathrm{MW}$ (Fig. $2 \mathrm{~d})$. As the current passes through the circuit's parasitic inductance, part of the electric energy is stored in the form of magnetic energy.
Consequently, when the capacitor's voltage reaches zero, the inductive energy sustains the current so that the capacitor's voltage becomes negative. The phase difference between the voltage and the current was approximately $90^{\circ}$ which arises from the low equivalent resistance of the discharge path. This was calculated to be $\sim 1.3 \mathrm{ohms}$, which includes equivalent resistance of the plasma and the wires.

Based on the numerous experiments performed, the peak current of the electric discharge becomes stable due to the low plasma impedance, and the shot-to-shot jitter of the delayed discharge current is measured to be less than $200 \mathrm{~ns}$.

Plasma Emission. Fig. 3 illustrates the typical integrated spectra of plasma generated from the metal samples. Several experiments are carried out to analyze each sample, i.e. LIBS at $10 \mathrm{~mJ}$ and $100 \mathrm{~mJ}$, and DB-LIBS and SA-LIBS at a laser pulse energy of $100 \mathrm{~mJ}$. Furthermore, the atomic and ionic characteristic lines of $\mathrm{Au}, \mathrm{Cu}, \mathrm{Ag}, \mathrm{Ni}, \mathrm{Al}$, and $\mathrm{Pd}$ are identified and labeled. The characteristic lines of nitrogen and hydrogen arise most likely from the background gas and humidity in the ambient atmosphere. The gate of the CCD of the spectrometer is set to be triggered at $1.5 \mu \mathrm{s}$ after the onset of the laser shot to minimize the background continuum emission, and the integration time is tuned to be $1.1 \mathrm{~ms}$. The observed background continuum emission is notably higher in the SA-LIBS spectra with respect to the other arrangements. In the case of LIBS at $100 \mathrm{~mJ}$, the spectral intensity is considerably higher than that of LIBS at $10 \mathrm{~mJ}$. In 

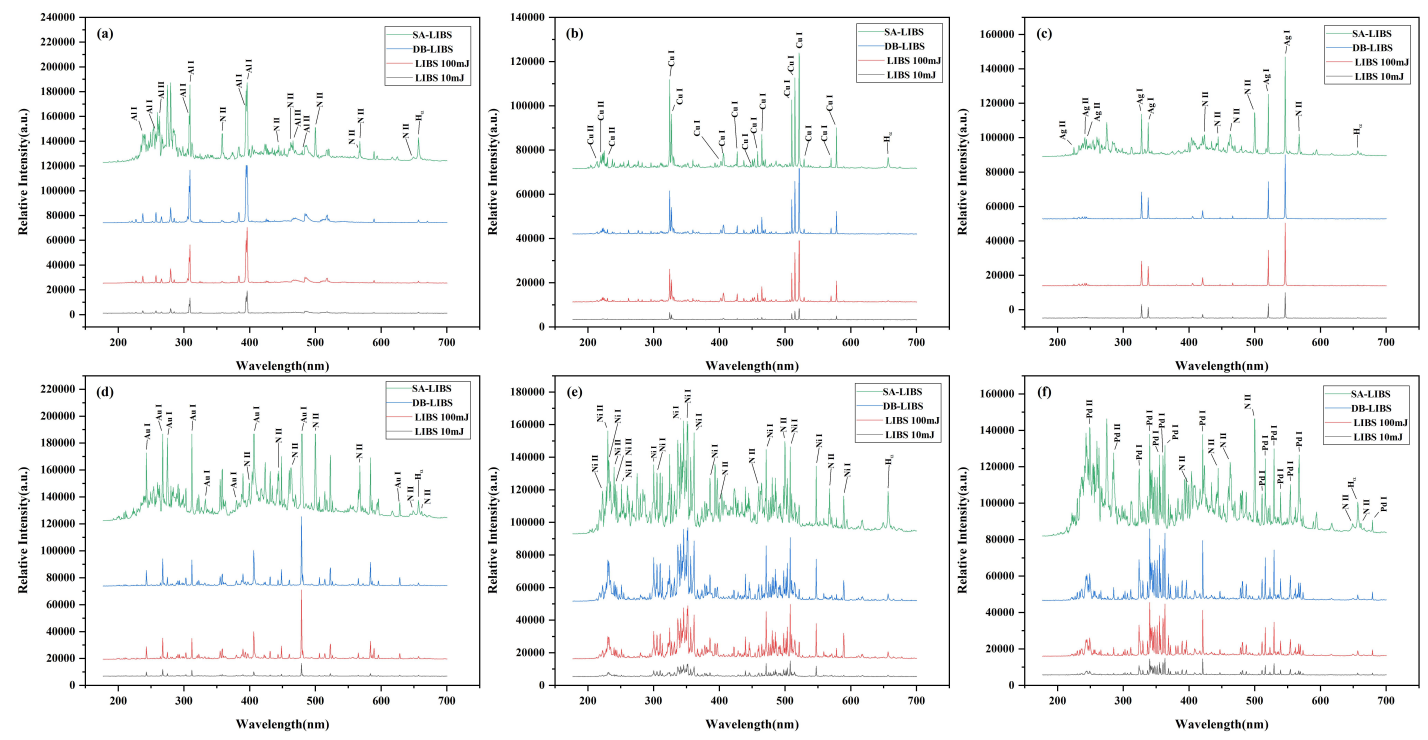

Fig. 3 Optical emission spectra of the LIBS at $10 \mathrm{~mJ}$ and $100 \mathrm{~mJ}$ per pulse, $\mathrm{CO}_{2}+\mathrm{LIBS}$, and SA-LIBS at $100 \mathrm{~mJ}$ per pulse for various targets (a) Al, (b) $\mathrm{Cu}$, and (c) Ag, (d) Au, (e) Ni, and (f) Pd. The spectra are shifted vertically for better clarity. CCD gate width: $1.1 \mathrm{~ms}$, spectrometer gate delay: $1.5 \mu$ s.

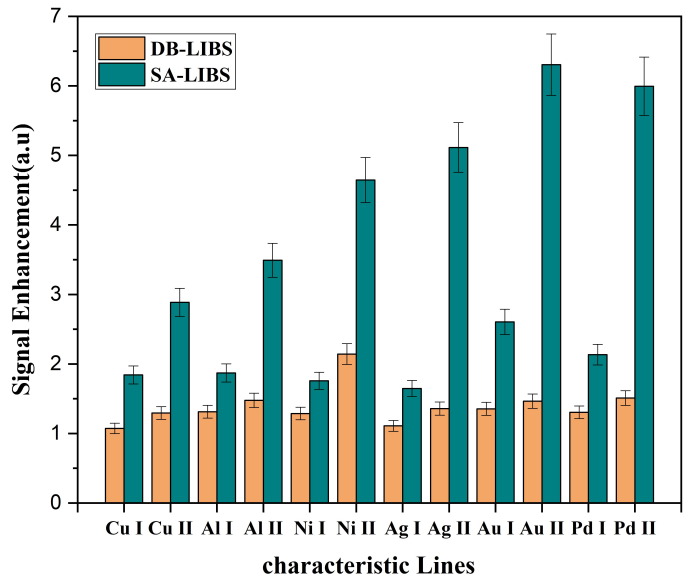

Fig. 4 Signal enhancement rate of atomic and ionic lines of the samples corresponding to DB-LIBS and SA-LIBS with respect to LIBS at a laser pulse energy of $100 \mathrm{~mJ}$.

fact, the larger number of atoms ablated from the sample surface contributes to extra photon emissions. Comparing the effects of $\mathrm{CW}-\mathrm{CO}_{2}$ laser and spark discharge, the spectra collected in SALIBS demonstrate more intensity enhancement. The plasma lifetime is obtained indirectly via changing the gate delay time of the spectrometer as long as the plasma emission could not be detected any longer. ${ }^{32}$ In SA-LIBS, the plasma lifetime is increased from $20 \mu \mathrm{s}$ to more than $120 \mu \mathrm{s}$. This provides more photons to be collected by the spectrometer, which results in spectral intensity enhancement. The signal enhancement follows the same trend for all metal samples. Because of employing a $30 \mathrm{~W} \mathrm{CW}-\mathrm{CO}_{2}$ laser and good heat conduction between the samples and the holder, the sample's temperature does not exceed $25^{\circ} \mathrm{C}$.

Fig. 4 illustrates the ratios of the atomic and ionic lines signal intensity in DB-LIBS and SA-LIBS with respect to the LIBS 100 $\mathrm{mJ}$ for a better assessment of the signal enhancement rate. The signal intensity is deduced by subtracting the continuum emission intensity at the signal's base from its peak intensity. As a result, the different background levels in the given spectrum are taken into account. The corresponding lines include $\mathrm{Cu}$ I at $521.6 \mathrm{~nm}, \mathrm{Cu}$ II at $229.4 \mathrm{~nm}, \mathrm{Au}$ I at $583.7 \mathrm{~nm}, \mathrm{Au}$ II at $299.4 \mathrm{~nm}, \mathrm{Ag} \mathrm{I}$ at 328.0 $\mathrm{nm}$, at $\mathrm{Ag}$ II at $241.9 \mathrm{~nm}, \mathrm{Pd}$ I at $421.2 \mathrm{~nm}, \mathrm{Pd}$ II at $229.6 \mathrm{~nm}, \mathrm{Al}$ I at $237.8 \mathrm{~nm}, \mathrm{Al}$ II at $281.6 \mathrm{~nm}, \mathrm{Ni}$ I at $324.3 \mathrm{~nm}$, and Ni II at 229.7 $\mathrm{nm}$. The error bars indicate the signal deviations corresponding to 10 repeated measurements. It is evident from the graph that the higher enhancement rates are attributed to the SA-LIBS spectra for both atomic and ionic lines. Furthermore, the ionic lines are more intensified against the atomic lines in DB-LIBS and SA-LIBS.

Plasma temperature, electron number density, and LTE. Plasma temperature is known as a significant parameter in the characterization of plasma processes. Regarding LTE approximation, the relationship of an optically thin transition between two levels of $E_{j}$ and $E_{i}$ of an atomic species and the corresponding emission is given by: ${ }^{33}$

$$
\frac{\lambda_{i j} I_{i j}}{h c A_{i j} g_{j}}=\frac{F C^{s}}{U(T)} e^{\left(\frac{E_{j}}{k T}\right)}
$$

where $I_{i j}$ and $\lambda_{i j}$ are the intensity and wavelength corresponding to transition from $i$ to $j$, respectively, $c$ and $h$ are the speed of light and the Planck's constant, $C^{s}$ and $k$ are the number density of the emitting species and the Boltzmann's constant, $A_{i j}$ is the transition probability between level $i$ and $j, F$ and $g_{j}$ are an experimental factor and the statistical weight of upper energy level $E_{j}$, and $U(T)$ is the partition function at the plasma temperature $\mathrm{T}$. Taking the natural logarithm on both sides, the 

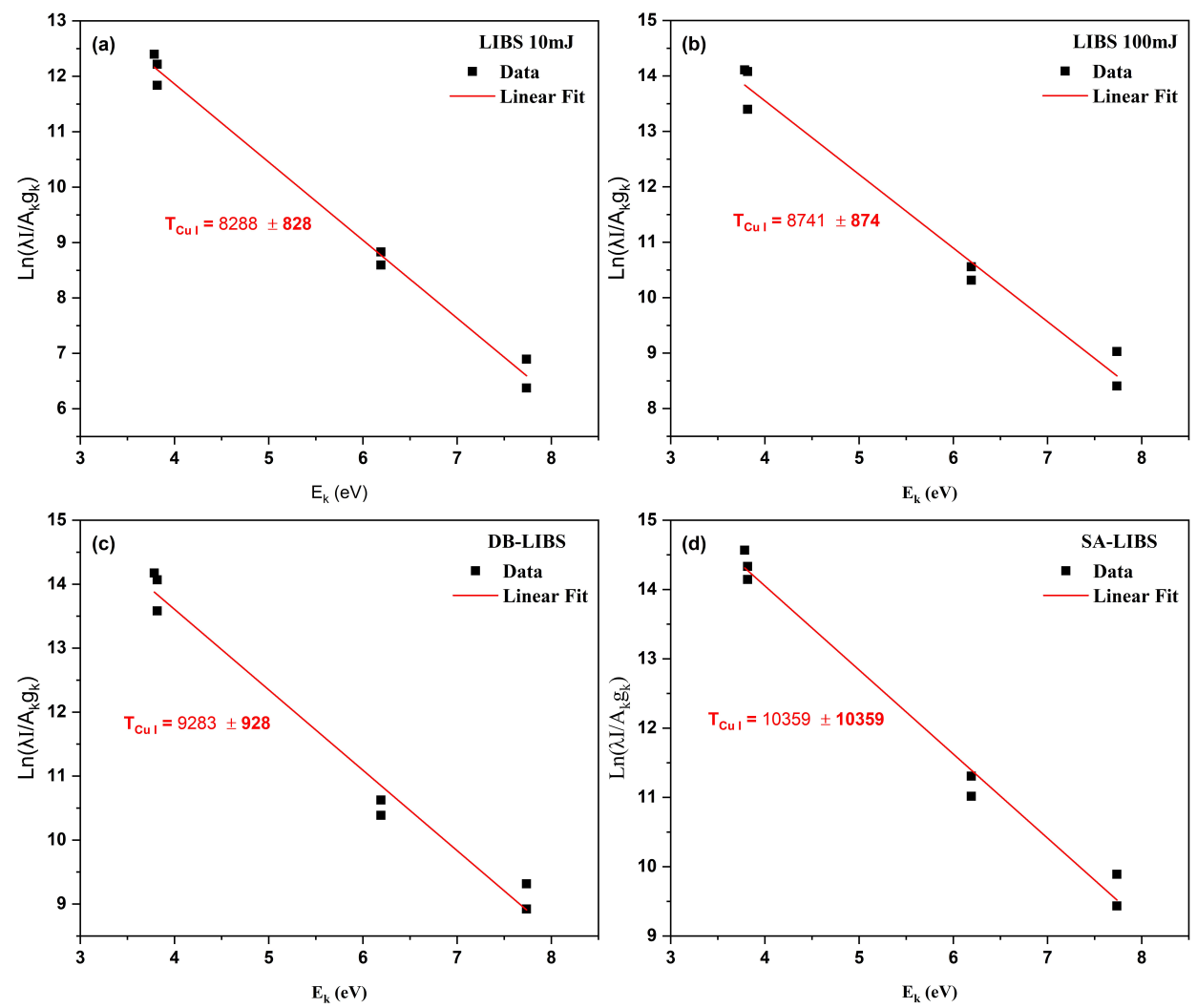

Fig. 5 Boltzmann plots and corresponding plasma temperature using emission lines of $\mathrm{Cu}$ I obtained from the spectra in (a) LIBS $10 \mathrm{~mJ}$, (b) LIBS 100 mJ, (c) DB-LIBS, and (d) SA-LIBS.

Table 1. The Wavelength, Upper-level Energy, Upper-level Degeneracy and Transition Probability for the Cu I Used for Plasma Temperature Calculation $^{34}$

\begin{tabular}{cccc}
\hline Atom/Ion & Wavelength(nm) & $\begin{array}{c}\text { Upper-level } \\
\text { energy(eV) }\end{array}$ & $\begin{array}{c}\mathbf{g}_{\mathbf{k}} \times \mathbf{A}_{\mathbf{k i}} \\
\left(\mathbf{1 0}^{\mathbf{7}} \mathbf{s}^{\mathbf{- 1}}\right)\end{array}$ \\
\hline $\mathbf{C u}$ I & 427.51 & 7.73 & 27.6 \\
$\mathbf{C u}$ I & 465.11 & 7.73 & 30.4 \\
$\mathbf{C u}$ I & 510.55 & 3.81 & 0.8 \\
$\mathbf{C u}$ I & 515.32 & 6.19 & 24 \\
$\mathbf{C u}$ I & 521.82 & 6.19 & 45 \\
$\mathbf{C u}$ I & 570.02 & 3.81 & 0.09 \\
$\mathbf{C u}$ I & 578.21 & 3.78 & 0.33 \\
\hline
\end{tabular}

Boltzmann plot equation is obtained as follows:

$$
\ln \left(\frac{\lambda_{i j} I_{i j}}{h c A_{i j} g_{j}}\right)=-\frac{1}{k T}\left(E_{j}\right)+\ln \left(\frac{F C^{s}}{U(T)}\right)
$$

when In $\left(\lambda_{i j} I_{i j} / h c A_{i j} g_{j}\right)$ versus $E_{j}$ is plotted, the electron temperature $T_{e}$ is determined based on the slope of the linear graph. The spectral parameters are collected from the database of the National Institute for Standards and Technology (NIST) ${ }^{34}$ To minimize the self-absorption effect, the lines corresponding to the transitions with energy levels $E_{i}$ under $1.4 \mathrm{eV}\left(11000 \mathrm{~cm}^{-1}\right)$, including the ground state, have been omitted. ${ }^{35}$ Hence, seven characteristic lines of $\mathrm{Cu} \mathrm{I}$ are examined for the plasma temperature calculations. The corresponding parameters of the atomic transitions used in this work are tabulated in Table 1.

Fig. 5 illustrates the typical Boltzmann plots and the corresponding plasma temperatures from $\mathrm{Cu}$ I transitions according to the spectra collected in a series of experiments during LIBS (10 mJ, $100 \mathrm{~mJ})$, DB-LIBS, and SA-LIBS. The RSD values of 10 repeated shots are obtained to be less than $6 \%$. Regarding the calculated temperatures, an increase in laser pulse energy from $10 \mathrm{~mJ}$ (Fig. 5a) to $100 \mathrm{~mJ}$ (Fig. 5b) does not necessarily lead to a remarkable plasma temperature rise. In fact, the higher laser pulse energy almost imparts in creating more ablated mass instead of heating the plasma. As a result, the relative signal intensity enhancement certainly occurs. Conversely, the plasma temperatures in DB-LIBS and SA-LIBS demonstrate to be lucidly higher than those of standard LIBS. In DB-LIBS (Fig. 5c), in which the plasma temperature is elevated $\sim 6 \%$, once a particle has evaporated, it starts absorbing the incoming coherent photons through the inverse Bremsstrahlung mechanism. The absorption cross-sections $\left(\mathrm{cm}^{2}\right)$ of the IB processes are given by: ${ }^{36}$

$$
\begin{gathered}
\sigma_{i b}(\lambda)_{\text {atom-electron }}=\frac{e^{2}}{\pi m_{e} c v^{2}} n_{e} n_{0} \sigma_{\text {coll }}\left(\frac{8 K_{B} T_{e}}{\pi m_{e}}\right) \\
\sigma_{i b}(\lambda)_{\text {ion-electron }}=\frac{3.7 \times 10^{18}}{T_{e}^{1 / 2} v^{3}} Z^{2}\left[\exp \left(\frac{h v}{K_{B} T_{e}}\right)-1\right] n_{e} n_{i} \quad \text { (Eq. 4) }
\end{gathered}
$$

where $n_{e}, n_{i}$, and $n_{o}$ are the electrons, ions, and neutral atoms number density and the electron density (in $\mathrm{cm}^{-3}$ ), respectively, $v$ is the laser irradiation frequency, $m_{e}$ and $\mathrm{Z}$ ascertain the electron mass and the ionic charge, and $\sigma_{\text {coll }}$ is the cross-section 
for electron-atom collision. It is evident that the absorption crosssections are strongly dependent on the laser wavelength $\left(\sigma_{i b}(\lambda)_{\text {atom-electron }} \propto \lambda^{2}\right)$. Note that in weakly ionized plasmas, the atom-electron processes are dominant. In fact, while IB absorption is significant at longer wavelengths $\left(10.6 \mu \mathrm{m}, \mathrm{CO}_{2}\right.$ laser), it notably reduces at shorter wavelengths $(1.064 \mu \mathrm{m}, \mathrm{Nd}$ : YAG laser). ${ }^{37}$ Supposing a laser-induced plasma lifetime of $\sim 20 \mu \mathrm{s}$, then a $30 \mathrm{~W}$ laser power would supply $\sim 0.6 \mathrm{~mJ}$ of additional energy compared to the $100 \mathrm{~mJ}$ Nd: YAG laser shot. However, regarding 10 times larger wavelength, the incident $\mathrm{CW}$ $\mathrm{CO}_{2}$ laser beam gives out $\sim 100$ times greater power absorption rate. Therefore, the contribution of the $\mathrm{CW}-\mathrm{CO}_{2}$ laser to plasma heating due to the IB process is quite considerable due to the IB process.

On the other hand, the electric discharge generates excessive high-energy electrons to reheat the plasma, leading to the higher temperatures. The properties of spark discharge plasmas are governed by various processes of electron generation and loss. Heating is a statistical process that transfers the directed energy obtained from the electric field into random energy of thermal motion. The total energy gained by electrons from the field is transferred to the gas due to the collisions with atoms and molecules. ${ }^{38}$ As a consequence, the average plasma temperature elevates $\sim 18 \%$ (Fig. $5 \mathrm{~d}$ ). The ionic to the atomic concentration ratio $n^{I I} / n^{I}$ can be obtained using the Saha-Eggert equation: ${ }^{39}$

$$
n_{e} \frac{n^{I I}}{n^{I}}=\frac{2}{n_{e}} \frac{\left(2 \pi k m_{e} k_{B} T\right)^{1.5}}{n^{3}} \frac{U^{I I}(T)}{U^{I}(T)} e^{-\frac{E_{i o n}}{k_{B} T}} \quad \text { (Eq. 5) }
$$

where $n^{I}$ and $n^{I I}$ ascertain the number densities of the neutral atomic and singly ionized species, respectively, $E_{\text {ion }}$ is the first ionization energy for an isolated system, and $m_{e}$ is the mass of the electron. Based on Eqs. (1 and 5), the ionized $I^{I I}$ to atomic $I^{I}$ line intensity ratio can be deduced as:

$$
\frac{I^{I I}}{I^{I}}=\frac{2}{n_{e}} \frac{\left(2 \pi k m_{e} k_{B} T\right)^{1.5}}{h^{3}} \frac{g^{I I} A^{I I} \lambda^{I}}{g^{I} A^{I} \lambda^{I I}} e^{-\frac{E^{I I}-E^{I}+E_{i o n}}{k_{B} T}} \quad \text { (Eq. 6) }
$$

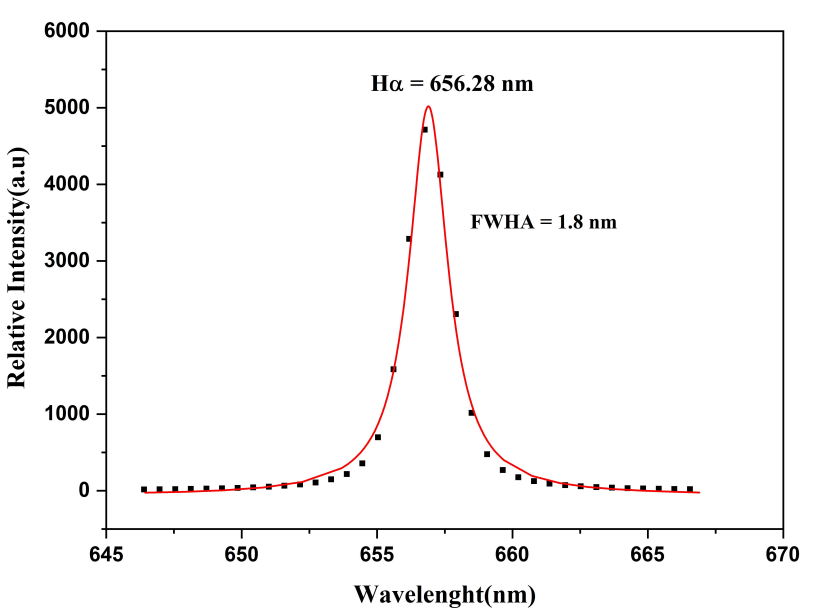

Fig. 6 Fitted Lorentzian profile of the $H_{\alpha}$ at $656.28 \mathrm{~nm}$ from the data collected in SA-LIBS.
Table 2. Typical Electron Density and Corresponding RSD Values Obtained Using $H_{\alpha}$ Line and Saha-Eggert Equation from the Cu Spectra During LIBS at $10 \mathrm{~mJ}$ and $100 \mathrm{~mJ}$, DB-LIBS and SA-LIBS at $100 \mathrm{~mJ}$

\begin{tabular}{lcccc}
\hline & \multicolumn{4}{c}{$\boldsymbol{N}_{\boldsymbol{e}}\left(\times \mathbf{1 0}^{\mathbf{1 7}} \mathbf{c m}^{-\mathbf{3}}\right)$} \\
\cline { 2 - 5 } Sample & Saha-Eggert & RSD $(\%)$ & $\boldsymbol{H}_{\boldsymbol{\alpha}}$ & RSD (\%) \\
\hline LIBS 10 mJ & 0.55 & 5 & --- & --- \\
LIBS 100 $\mathbf{~ J J}$ & 1.24 & 5.3 & 1.12 & 4.7 \\
DB-LIBS 100 mJ & 2.84 & 4.3 & 2.68 & 4.2 \\
SA-LIBS 100 mJ & 9.3 & 6.8 & 7.9 & 6.3 \\
\hline
\end{tabular}

According to the above equation, it can be seen that an increase in plasma temperature promotes the ionization degree such that the upper levels become more populated, and this addresses the appearance of more intense ionic lines in DB-LIBS and SALIBS. $^{40}$ In addition, part of the metastable nitrogen atoms' energy may also enhance the spectral intensity.

The electron number density is an equally vital parameter in order to study the degree of thermodynamic equilibrium in laserinduced plasmas. Its value plays a crucial role in estimating the LTE departure, which is a critical condition for employing the calibration-free technique. The $n_{e}$ can be obtained from Eq. (6) once an ionized and an atomic line intensity besides the plasma temperature are predetermined. Furthermore, the measurement of the Stark broadened $H_{\alpha}$ line profile (originated from atmospheric humidity) is recognized as a reliable method to estimate $n_{e}$ in laser-induced plasmas. ${ }^{41}$ It is worth mentioning that in SA-LIBS, the substantial Stark broadening of the hydrogen lines provides sufficiently accurate linewidth measurement, even using a lowresolution spectrometer. The Doppler broadening is calculated to be less than $0.02 \mathrm{~nm}$ and the instrumental broadening $\Delta \lambda_{\text {ins }}$ is determined to be $0.35 \mathrm{~nm}$ by measuring the spectral broadening of the $\mathrm{Hg}$ line at $546.07 \mathrm{~nm}$ emitted from a low-pressure mercury lamp. After subtracting $\Delta \lambda_{\text {ins }}$ from the observed line profile, the electron number density is calculated via the full width at half area (FWHA) to be much less sensitive to ion dynamics effects than the usual full width at half maximum (FWHM) according to the following relation: ${ }^{42}$

$$
H_{\alpha}: F W H A=0.549 \mathrm{~nm} \times 10^{12}\left(\frac{N_{e}}{10^{23} m^{-3}}\right)^{0.67965} \text { (Eq. 7) }
$$

Fig. 6 depicts the Lorentz fitted profile of the Stark broadened $H_{\alpha}$ lines during a typical SA-LIBS experiment. The typical electron densities obtained from the spectra in LIBS $(10 \mathrm{~mJ}$, $100 \mathrm{~mJ}$ ), SA-LIBS, and DB-LIBS are tabulated in Table 2. The calculated electron density values from both methods are in good agreement. The corresponding RSD values in 10 repeated shots were determined to be less than $7 \%$. It is apparent that the electron number density elevates 2 times as laser pulse energy increases from $10 \mathrm{~mJ}$ to $100 \mathrm{~mJ}$ in standard LIBS. The CW$\mathrm{CO}_{2}$ laser reheats the plasma leading to a further increase of $n_{e}$ (2 times), whereas in SA-LIBS, the average electron density elevates up to one order of magnitude. 
The LTE is a state in which the temperature of all species is assumed to be the same. In order to assess the LTE, three criteria are investigated in this work. The McWhirter criterion is a condition to ensure that collisional events dominate over radiative processes such that the deviation from LTE becomes negligible. It is given by: ${ }^{43}$

$$
N_{e}>1.6 \times 10^{12} \times \Delta E^{3} \times T^{0.5}(\text { Eq. } 8)
$$

where $\Delta \mathrm{E}$ is the highest energy value of the corresponding electronic transition. The right-hand expression in Eq. (7) is calculated for $\mathrm{Cu}(\mathrm{I})$ at $276.63 \mathrm{~nm}$, which give out $\sim 1.2 \times$ $10^{16} \mathrm{~cm}^{-3}$, much lower than the smallest value of $n_{e}$ obtained in this work $\sim 5.5 \times 10^{16} \mathrm{~cm}^{-3}$. As a result, due to larger electron number densities, SA-LIBS satisfies the McWhirter criterion more confidently than DB-LIBS and standard LIBS. In the case of inhomogeneous and transient plasmas, two more criteria are proposed by Cristoforetti et al. ${ }^{44}$ to allow for a more careful assessment of the LTE condition. The first one indicates that the diffusion length $\bar{\lambda}$ of the atoms/ions should be shorter than the variation length of the plasma temperature and the electron number density during the relaxation time. The second one deals with the time to reach excitation and ionization equilibrium $\tau_{\text {relax }}$, which should be much shorter than the variation time of the thermodynamic parameters in the plasma. Here, the value of $\tau_{\text {relax }}$ and $\bar{\lambda}$ is estimated using $\mathrm{Cu}(\mathrm{I})$ resonant transitions based on the Eqs. (13) and (15) in Ref 43. Considering a plasma diameter $d$ of $\sim 2 \mathrm{~mm}$, the longest diffusion length is determined to be $\sim 3 \mu \mathrm{m}$ so that criterion $\bar{\lambda} \ll$ $d$ is well fulfilled. Subsequently, a maximum value of $\tau_{\text {relax }} \sim 150$ ps was obtained, which is considerably shorter than the plasma expansion time of $\sim 1 \mu \mathrm{s}$. Therefore, the plasma enjoys the LTE conditions since all of the above criteria are well satisfied.

\section{CONCLUSIONS}

This work deals with the identification of different targets, i.e. $\mathrm{Cu}$, $\mathrm{Au}, \mathrm{Ag}, \mathrm{Al}, \mathrm{Ni}$, and $\mathrm{Pd}$ samples, using various methods, including LIBS, DB-LIBS, and SA-LIBS. The spectral intensity enhancement is systematically investigated based on $\mathrm{CW}-\mathrm{CO}_{2}$ laser and spark discharge assisted LIBS. It was found that the use of a low power laser at a longer wavelength $(10.6 \mu \mathrm{m})$ can effectively reheat the plasma through the inverse Bremsstrahlung mechanism leading to $\sim 6 \%$ temperature rise. In SA-LIBS, besides the elevated plasma temperature of $\sim 18 \%$, the extended lifetime plays a significant role in signal intensification. Subsequently, the rise of plasma temperature promotes the ionization degree such that the upper levels get more populated. As a consequence, more intense ionic lines appear in the spectra for all metal specimens. In addition, a remarkable increase of electron number density occurs in SA-LIBS, around one order of magnitude, where LTE firmly holds. Eventually, it is expected that the low-cost SA-LIBS will act as a better technique against DBLIBS, in addition to the ability to incorporate it into portable devices for field analyses.

\section{AUTHOR INFORMATION}

\section{Corresponding Author}

* P. Parvin

Email address: parvin@ @ut.ac.ir

\section{Notes}

The authors declare no competing financial interest.

\section{ACKNOWLEDGMENTS}

We are thankful to Fatemeh Shahi for her collaboration and useful discussion.

\section{REFERENCES}

1. D. W. Hahn and N. Omenetto, Appl. Spectrosc., 2010, 64, 335A-366A. https://doi.org/10.1366\%2F000370210793561691

2. D. W. Hahn and N. Omenetto, Appl. Spectrosc., 2012, 66, 347-419. https://doi.org/10.1366\%2F11-06574

3. J. Rakovský, P. Čermák, O. Musset, and P. Veis, Spectrochim. Acta B, 2014, 101, 269-287. https://doi.org/10.1016/j.sab.2014.09.015

4. L. Balika, C. Focsa, S. Gurlui, S. Pellerin, N. Pellerin, D. Pagnon, and M. Dudeck, Spectrochim. Acta B, 2012, 74, 184-189. https://doi.org/10.1016/j.sab.2012.06.030

5. S. Schröder, S. G. Pavlov, I. Rauschenbach, E. K. Jessberger, and H. W. Hübers, Icarus., 2013, 223, 61-73. https://doi.org/10.1016/j.icarus.2012.11.011

6. A. Ciucci, V. Palleschi, S. Rastelli, A. Salvetti, D. P. Singh, and E. Tognoni, Laser Part. Beams., 1999, 17, 793-797. https://doi.org/10.1017/S0263034699174251

7. B. Praher, V. Palleschi, R. Viskup, J. Heitz, and J. D. Pedarnig, Spectrochim. Acta B, 2010, 65, 671-679. https://doi.org/10.1016/j.sab.2010.03.010

8. M. D. Assefa, A. K. Chaubey, A. T. Hailu, and A. Y. Hibstie, Eur. Phys. J. Appl. Phys., 2013, 62, 30701. https://doi.org/10.1051/epjap/2013120525

9. V. S. Burakov, V. V. Kiris, P. A. Naumenkov, and S. N. Raikov, J. Appl. Spectrosc., 2004, 71, 740-746. https://doi.org/10.1023/B:JAPS.0000049638.55151.80

10. K. K. Herrera, E. Tognoni, N. Omenetto, B. W. Smith, and J. D. Winefordner, J. Anal. At. Spectrom., 2009, 24, 413-425. https://doi.org/10.1039/b820493d

11. P. J. Kolmhofer, S. Eschlböck-Fuchs, N. Huber, R. Rössler, J. Heitz, and J. D. Pedarnig, Spectrochim. Acta B, 2015, 106, 67-74. https://doi.org/10.1016/j.sab.2015.02.004

12. M. Jandaghi, P. Parvin, M. J. Torkamany, and J. Sabbaghzadeh, J. Phys. D., 2009, 42, 205301. https://doi.org/10.1088/0022-3727/42/20/205301 
13. M. Corsi, G. Cristoforetti, M. Hidalgo, S. Legnaioli, V. Palleschi, A. Salvetti, E. Tognoni, and C. Vallebona, Appl. Geochem., 2006, 21, 748-755. https://doi.org/10.1016/j.apgeochem.2006.02.004

14. L. I. Kexue, W. Zhou, and Q. Shen, Z. Ren, and B. Peng, J. Anal. At. Spectrom., 2010, 25, 1475-1481. https://doi.org/10.1039/B922187E

15. A. M. Popov, F. Colao, and R. Fantoni, J. Anal. At. Spectrom., 2009, 24, 602-604. https://doi.org/10.1039/B818849A

16. A. Hussain, Q. Li, Z. Hao, X. Gao, and J. Lin, Plasma Sci. Technol., 2015, 17, 693-698. https://doi.org/10.1088/1009-0630/17/8/14

17. S. M. Angel, D. N. Stratis, K. L. Eland, T. Lai, M. A. Berg, and D. M. Gold, J. Anal. Chem., 2001, 369, 320-327. https://doi.org/10.1007/s002160000656

18. J. Skruibis, O. Balachninaite, S. Butkus, V. Vaicaitis, and V. Sirutkaitis, Opt. Laser Technol., 2019, 111, 295-302. https://doi.org/10.1016/j.optlastec.2018.10.005

19. Y. Liu, B. Bousquet, M. Baudelet, and M. Richardson, Spectrochim. Acta B, 2012, 73, 89-92. https://doi.org/10.1016/j.sab.2012.06.041

20. K. A. Tereszchuk, J. M. Vadillo, and J. J. Laserna, Appl. Spectrosc., 2008, 62, 1262-1267. https://doi.org/10.1366/000370208786401491

21. W. Zhou, L. I. Kexue, Q. Shen, and J. Long, Opt. Express., 2010, 18, 2573-2578. https://doi.org/10.1364/OE.18.002573

22. S. Laville, C. Goueguel, H. Loudyi, F. Vidal, M. Chaker, and M. Sabsabi, Spectrochim. Acta B, 2009, 64, 347-353. https://doi.org/10.1016/j.sab.2009.03.021

23. C. Goueguel, S. Laville, F. Vidal, M. Sabsabi, and M. Chaker, J. Anal. At. Spectrom., 2010, 25, 635-644. https://doi.org/10.1039/B927013B

24. Y. C. Li; D. Tian, Y. Ding, G. Yang, K. Liu, C. H. Wang, and X. Han, Appl. Spectrosc. Rev., 2018, 53, 1-35. https://doi.org/10.1080/05704928.2017.1352509

25. D. K. Killinger, S. D. Allen, R. D. Waterbury, C. Stefano, and E. L. Dottery, Opt. Express., 2007, 15, 12905-12915. https://doi.org/10.1364/OE.15.012905

26. S. Z. Shoursheini, P. Parvin, B. Sajad, and M. A. Bassam, Appl. Spectrosc., 2009, 63, 423-429. https://doi.org/10.1366/000370209787944262

27. O. A. Nassef and H. E. Elsayed-Ali, Spectrochim. Acta B, 2005, 60, 1564-1572. https://doi.org/10.1016/j.sab.2005.10.010
28. W.-D. Zhou, Y.-H. Guo, and R.-R. Zhang, Front. Phys., 2020, 15, 52201. https://doi.org/10.1007/s11467-020-0969-1

29. J. Kang, Y. Chen, and R. Li, Spectrochim. Acta B, 2019, 161, 105711. https://doi.org/10.1016/j.sab.2019.105711

30. J. Gao, J. Kang, R. Li, and Y. Chen, Appl. Opt., 2020, 59, 4091-4096. https://doi.org/10.1364/AO.390723

31. M. Habibpour, P. Parvin, R. Amrollahi, Appl. Opt., 2021, 60, 1099-1109. https://doi.org/10.1364/AO.416032

32. S. Z. Mortazavi, P. Parvin, M. R. Mousavi Pour, A. Reyhani, A. Moosakhani, and S. Moradkhani, Opt. Laser Technol., 2014, 62, 32-39. https://doi.org/10.1016/j.optlastec.2014.02.006

33. A. Moosakhani, P. Parvin, A. Reyhani, and S. Z. Mortazavi, Phys. Plasmas, 2017, 24, 013505. https://doi.org/10.1063/1.4973554

34. National Institute of Standards and Technology, "Electronic database," https://www.nist.gov/pml/atomic spectra-database.

35. A. De Giacomo, M. Dell'Aglio, O. De Pascale, S. Longo, and M. Capitelli, Spectrochim. Acta B, 2007, 62, 1606-1611. https://doi.org/10.1016/j.sab.2007.10.004

36. L. Fornarini, F. Colao, R. Fantoni, V. Lazic, and V. Spizzicchino, Spectrochim. Acta B, 2005, 60, 1186-1201. https://doi.org/10.1016/j.sab.2005.06.008

37. R. W. Coons, S.S. Harilal, S.M. Hassan, and A. Hassanein, Appl. Phys. B., 2012, 107, 873-880. https://doi.org/10.1007/s00340-012-4997-6

38. Y. P. Raizer and J. E Allen - 1997. Vol. 2. Berlin: Springer.

39. J. A. M. Van der Mullen, Phys. Rep., 1990, 191, 109-220. https://doi.org/10.1016/0370-1573(90)90152-R

40. H. Sobral, G. Quintana-Silva, and A. Robledo-Martinez, Spectrochim. Acta B, 2020, 167, 105844. https://doi.org/10.1016/j.sab.2020.105844

41. C. Aragón and J.A. Aguilera, Spectrochim. Acta B, 2010, 65, 395-400. https://doi.org/10.1016/j.sab.2010.03.020

42. M. A. Gigosos, M. Á. González, and V. Cardeñoso, Spectrochim. Acta B, 2003, 58, 1489-1504. https://doi.org/10.1016/S0584-8547(03)00097-1

43. G. Cristoforetti, E. Tognoni, and L. A. Gizzi, Spectrochim. Acta B, 2013, 90, 1-22. https://doi.org/10.1016/j.sab.2013.09.004

44. G. Cristoforetti, A. De Giacomo, M. Dell'Aglio, S. Legnaioli, E. Tognoni, V. Palleschi, and N. Omenetto, Spectrochim. Acta B, 2010, 65, 86-95. https://doi.org/10.1016/j.sab.2009.11.005 